\title{
Efeitos da Pulsação Ultrassônica da Corrente sobre a Geometria da ZF e ZAC na Soldagem Arco Submerso
}

\author{
Anna Louise Voigt ${ }^{1}$, Tiago Vieira da Cunha ${ }^{1}$ \\ 1 Universidade Federal de Santa Catarina - UFSC, Centro de Engenharias da Mobilidade, Joinville, SC, Brasil.
}

Recebido: 26 Abr., 2016

Aceito: 15 Ago., 2016

E-mails: alouisevoigt@gmail.com (ALV), t.cunha@ufsc.br (TVC)
Resumo: O presente trabalho propõe investigar a aplicação da técnica de excitação ultrassônica do arco no processo SAW com o intuito de avaliar a influência dos principais parâmetros associados a pulsação da corrente, como a frequência e a amplitude da corrente de excitação ultrassônica, sobre os cordões de solda produzidos. Para tanto, foi montada uma bancada experimental constituída por duas fontes de energia: uma convencional de soldagem e outra capaz de pulsar a corrente em frequências ultrassônicas. Durante a realização dos ensaios foram empregadas duas amplitudes de corrente de excitação ultrassônica, 25 A e 50 A, cada qual em três frequências de pulsação diferentes, $20 \mathrm{kHz}, 50 \mathrm{kHz}$ e $80 \mathrm{kHz}$. A partir de macrografias foram realizadas análises dimensionais dos cordões obtidos com foco na largura, penetração e área fundida, assim como a área da zona afetada pelo calor (ZAC). Como resultado, contrariando as informações disponíveis na literatura, não foi possível identificar alterações consideráveis na geometria do cordão de solda, assim como, na ZAC que pudessem ser atribuídas à pulsação da corrente nas frequências estudadas.

Palavras-chave: Arco submerso; Pulsação da corrente; Frequências ultrassônicas; Geometria da zona fundida; Zona termicamente afetada.

\section{Research on Effects of Arc Excited Ultrasonic on Fusion and Heat Affected Zone in Submerged Arc Welding}

\begin{abstract}
This paper proposes an investigation of arc ultrasonic excitation technique in SAW process, in order to evaluate the main parameters influence associated with pulsed current on weld beads, such as the frequency and amplitude of ultrasonic excitation current. Therefore, an experimental bench was assembled, which consists of two power sources: a conventional welding source and another able to pulse current at ultrasonic frequencies. During the tests were employed two ultrasonic excitation current amplitudes, $25 \mathrm{~A}$ and $50 \mathrm{~A}$, each one in three different pulse frequencies, $20 \mathrm{kHz}, 50 \mathrm{kHz}$ and $80 \mathrm{kHz}$. Macrographs of these welds are observed, and width, penetration, molten area and heat affected zone area (HAZ) are measured. As a result, contrary to the information available in literature, there were no significant changes in weld bead geometry, as well as in the HAZ, that could be attributed to the current excitation in studied frequencies.
\end{abstract}

Key-words: Submerged arc welding; Ultrasonic arc excitation; Ultrasonic frequencies; Molten pool geometry; Heat affected zone.

\section{Introdução}

Ao longo das últimas décadas, diversas técnicas têm sido estudadas com o intuito de melhorar a produtividade e a qualidade dos cordões de solda produzidos pelos mais diversos processos de soldagem a arco. Neste contexto, no final da década de 1990, Wu et al. [1] propuseram uma nova técnica de soldagem. Trata-se do arco com excitação ultrassônica da corrente, onde o arco atua tanto como fonte de calor, como também, um mecanismo de emissão de ultrassom. $\mathrm{O}$ arco com excitação ultrassônica é obtido através de uma corrente principal de soldagem e uma corrente pulsada com frequência de pulsação superior a $20 \mathrm{kHz}$. Para tanto são conectadas duas fontes em paralelo sendo a fonte convencional responsável pelo fornecimento da corrente principal de soldagem e uma fonte de excitação capaz de pulsar a corrente em frequências ultrassônicas. É possível encontrar na literatura a aplicação desta técnica em processos como plasma melt-in [2], plasma key-hole [3], TIG [4], eletrodo revestido [5], inclusive no arco submerso. Em relação a este último, melhoras tanto na microestrutura quanto nas propriedades mecânicas das juntas soldadas são atribuídas à aplicação da citada técnica. Conforme relatado por
Este é um artigo publicado em acesso aberto (Open Access)
sob a licença Creative Commons Attribution Non-Commerci que permite uso, distribuição e reprodução em qualquer meio, sem restrições des de que sem fins comerciais e que 0 trabalho original seja corretamente citado. 
Yang et al. [6], além do refino de grão obtido na zona fundida, ensaios de impacto a baixa temperatura mostram que o arco excitado com ultrassom pode notavelmente melhorar a resistência ao impacto na zona fundida. Relativo a isto, Wu et al. [7] obtiveram uma melhora em torno de 50\% tanto na zona fundida quanto na ZAC, ao realizar ensaios de impacto a uma temperatura de -70 으 nos corpos de prova do aço GBT 09MnNiDR soldados com frequência de pulsação de $50 \mathrm{kHz}$ no processo SAW. Utilizando como material de base um aço com baixo teor de carbono, Zhang et al. [8] observaram que a microestrutura da zona fundida mudou consideravelmente de ferrita lamelar para acicular ao se empregar a pulsação da corrente em frequências ultrassônicas. Os cordões com excitação ultrassônica apresentaram mais ferrita acicular, enquanto que nos cordões sem excitação foi observada mais ferrita de placas laterais. Visto que a ferrita de placas laterais se forma em temperaturas superiores à ferrita acicular, os autores concluem, portanto, que o arco com excitação ultrassônica acelera o resfriamento do metal na zona fundida e, consequentemente, a transferência de calor na ZAC. Segundo Zhang et al. [8], isto ocorre devido à vibração ultrassônica, pois o ultrassom se propaga na região da ZAC, que se expande e comprime periodicamente levando a mudança na densidade da matriz. De acordo com os autores, na compressão, a temperatura aumenta devido à diminuição do volume e o inverso ocorre na expansão. Na matriz, este gradiente de temperatura entre áreas em compressão e em expansão faz com que o calor transferido flua da área de maior para a de menor temperatura. Segundo Zhang et al. [8], esse processo acelera a transferência de calor e altera a distribuição de temperatura levando a um aumento do tamanho da ZAC. Em seus cordões, Zhang et al. [8] obtiveram um aumento expressivo da zona afetada pelo calor (ZAC) com o aumento da frequência de pulsação ultrassônica, conforme pode ser visto na Figura 1, para uma corrente de soldagem de aproximadamente $500 \mathrm{~A}$ e uma tensão de arco de $30 \mathrm{~V}$. Os autores comentam que há uma melhora das propriedades da ZAC, contudo, sem descrever quais são as propriedades.

Por se tratar de uma técnica relativamente nova, proposta a pouco mais de uma década, há ainda certa carência de estudos que permitam compreender, de fato, as implicações quanto a sua utilização. Apesar de ser possível encontrar na literatura trabalhos cujos resultados evidenciam, conforme citado anteriormente, o seu

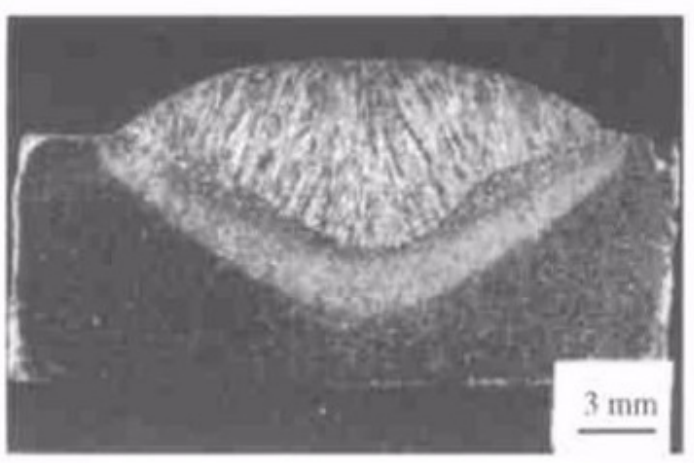

(a) Sem excitação

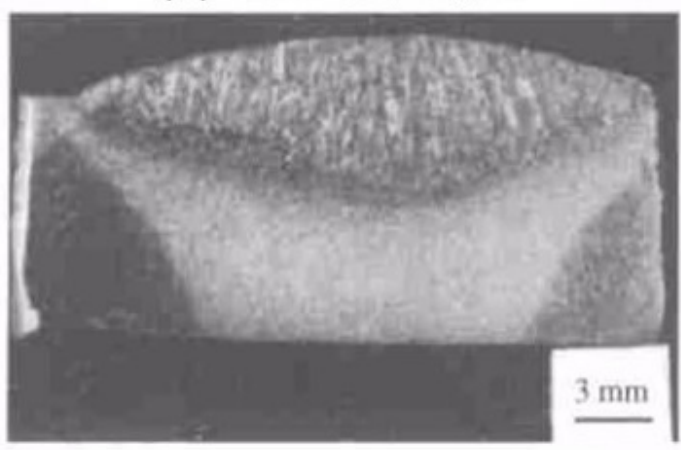

(c) Excitação de $50 \mathrm{kHz}$

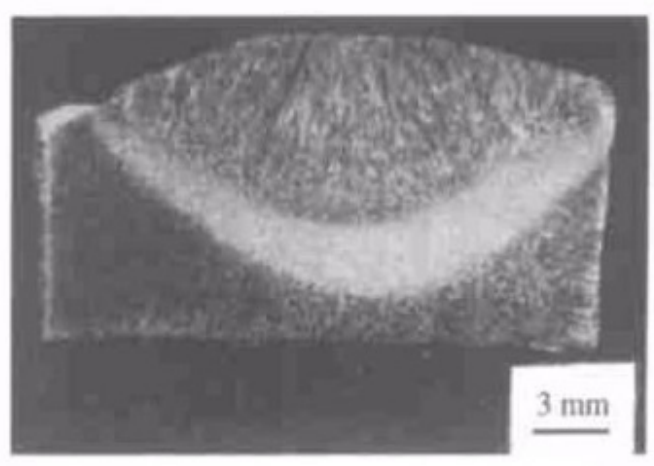

(b) Excitação de $30 \mathrm{kHz}$

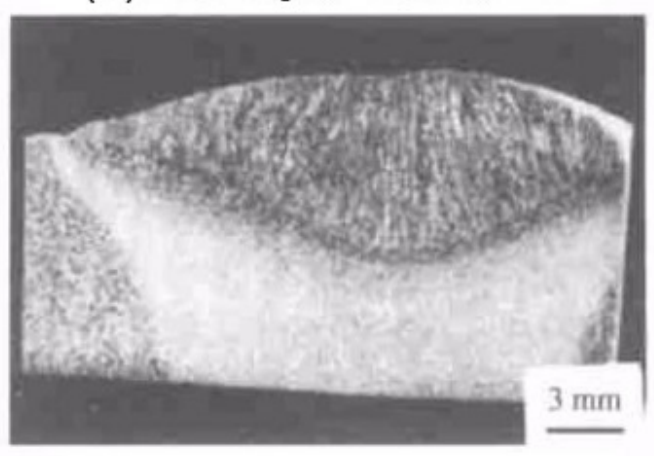

(d) Excitação de $80 \mathrm{kHz}$

Figura 1. Macrografias dos cordões obtidos por Zhang et al. [8] com diferentes frequências de pulsação da corrente. 
potencial, estes em sua maioria são de origem chinesa, fato que dificulta o entendimento integral dos efeitos da aplicação da técnica. Em vista disso, o presente trabalho tem, portanto, como objetivo realizar uma investigação no que diz respeito à aplicação da técnica de excitação ultrassônica do arco no processo arco submerso, tema esse, ainda incipiente no Brasil.

\section{Materiais e Métodos}

De modo a avaliar os efeitos da aplicação da técnica de excitação ultrassônica do arco no processo arco submerso, foram realizados cordões de solda de simples deposição sobre chapa (bead-on-plate). Para a realização dos ensaios, foi concebida uma bancada composta por uma fonte de soldagem multiprocesso, modelo DigiPLUS A7, com capacidade de corrente de até $800 \mathrm{~A}$ e a fonte de soldagem desenvolvida por Cunha [9] responsável pelo fornecimento da corrente de excitação ultrassônica, ambas conectadas em paralelo. Esta última permite obter correntes pulsadas com diversas formas de onda, em frequências ultrassônicas, a qual o autor usou numa primeira aplicação para o processo TIG, porém, a mesma permite aplicação em outros processos. Esta fonte de soldagem possui capacidade de fornecimento de corrente de excitação ultrassônica com frequências de pulsação $\left(f_{\text {UIT }}\right)$ de $20 \mathrm{kHz}$ a $80 \mathrm{kHz}$ com amplitudes de pico a pico de até $50 \mathrm{~A}\left(\mathrm{I}_{\mathrm{ULT}}\right)$. A fonte principal foi configurada para operar no modo tensão constante, de modo a manter constante a tensão de arco de acordo com o valor ajustado no equipamento (auto-ajuste). Já a fonte de excitação ultrassônica opera no modo corrente constante, a fim de se conseguir a adequada pulsação da corrente. Nesta, por se tratar de uma fonte de corrente contínua, foi ajustado um valor de offset da corrente de $30 \mathrm{~A}$, a fim de permitir a pulsação da corrente com amplitude de pico a pico de até $50 \mathrm{~A}$, como ilustrado na Figura 2. Este valor de $30 \mathrm{~A}$, fornecido pela fonte de excitação, juntamente com a corrente principal de soldagem $\left(I_{P R I}\right)$, resulta em $I_{M E D}$ Isso porque a fonte convencional desconta o valor médio da corrente ultrassônica por se tratar de uma fonte do tipo tensão constante onde, portanto, o auto ajuste da corrente está presente. Deste modo, independente do valor médio da corrente ultrassônica, o valor médio da corrente de soldagem $\left(\mathrm{I}_{\mathrm{PRI}}+\mathrm{I}_{\mathrm{ULT}}\right)$ será sempre o mesmo devido a este auto controle.

Para a movimentação do cabeçote de soldagem desenvolvido para este trabalho foi utilizado um sistema de deslocamento dotado de um grau de liberdade - Tartílope V1. A bancada montada e alguns dos seus itens descritos é mostrada na Figura 3.

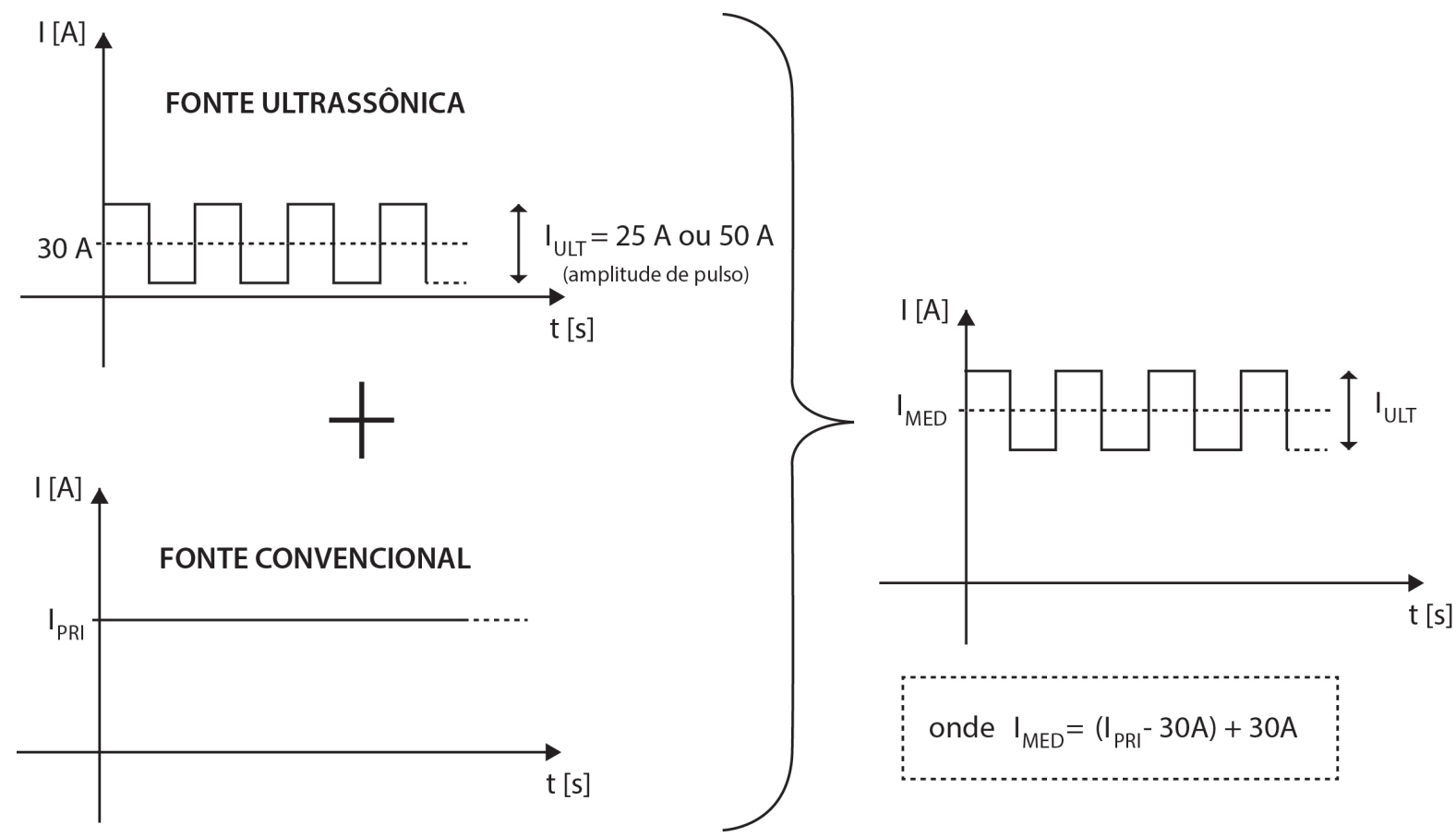

Figura 2. Funcionamento da corrente das fontes conectadas em paralelo. 


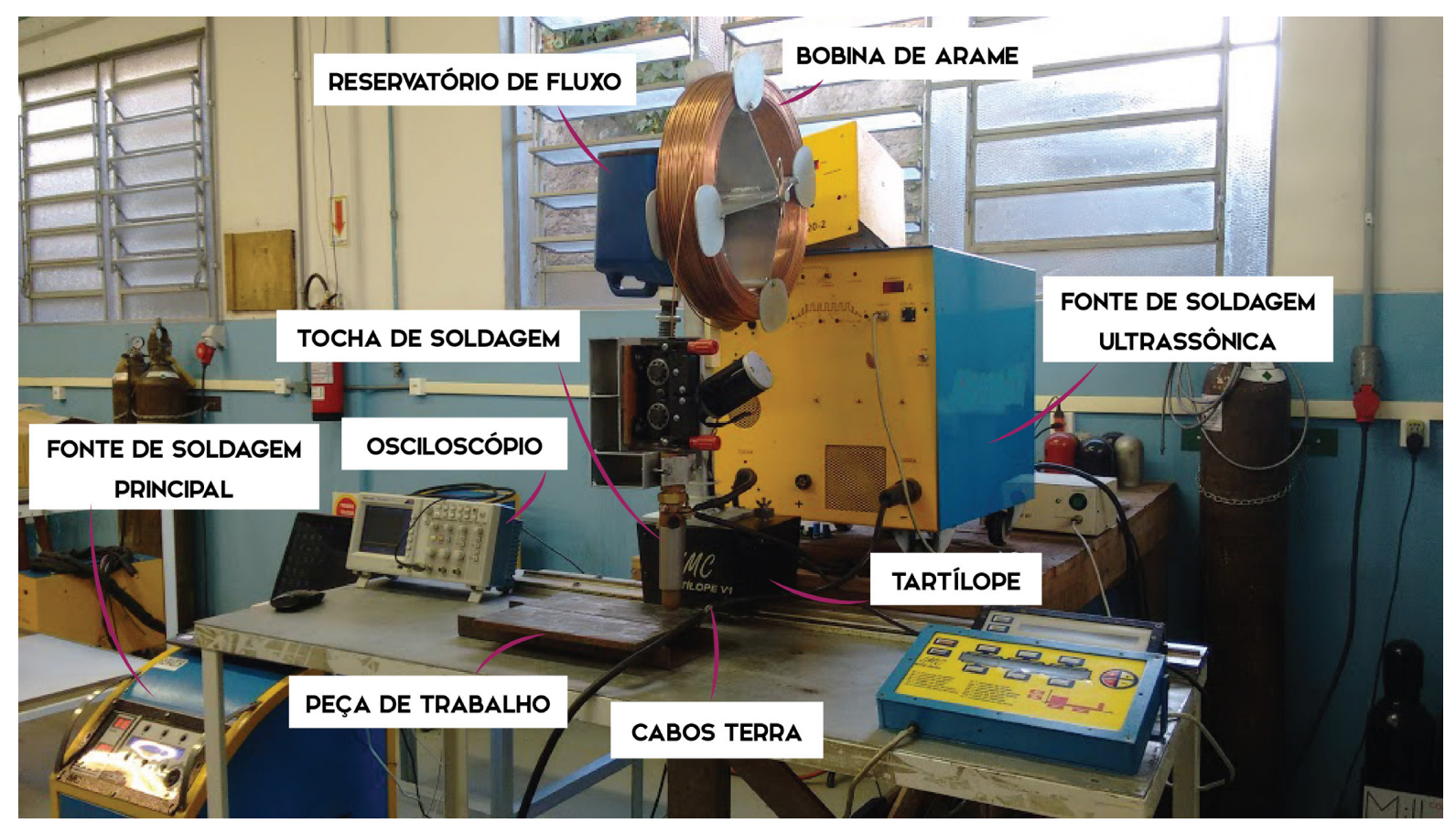

Figura 3. Bancada de ensaios.

Para realização dos ensaios, foram utilizados corpos de prova de 300×110x10 mm de aço ASTM A36. O metal de adição utilizado foi o arame maciço de aço-carbono com diâmetro de 2,4 mm, classificação EM12K segundo a AWS 5.17, e o conjunto arame-fluxo utilizado nos ensaios foi o AWS F7P6-EM12K. Os demais parâmetros de soldagem como tensão de arco, velocidade de alimentação de arame e distância entre bico contato-peça (DBCP) foram fixados em todos os ensaios e seus valores se encontram na Tabela 1.

Tabela 1. Parâmetros empregados em todos os ensaios.

\begin{tabular}{ll}
\hline \multicolumn{1}{c}{$\quad$ Parâmetro } & Valor \\
Tensão de arco & $30 \mathrm{~V}$ \\
DBCP & $20 \mathrm{~mm}$ \\
Velocidade de soldagem & $60 \mathrm{~cm} / \mathrm{min}$ \\
Velocidade de alimentação de arame & $2,5 \mathrm{~m} / \mathrm{min}$ \\
Diâmetro do arame & $2,4 \mathrm{~mm}$ \\
\hline
\end{tabular}

A fim de atender aos objetivos do trabalho foram realizados seis diferentes cordões de solda. Para analisar os possíveis efeitos da frequência de pulsação da corrente de excitação, foram realizados ensaios com as frequências de 20, 50 e 80 kHz, pois, de acordo com a literatura [10], são as frequências em que os efeitos da pulsação ultrassônica são mais expressivos. Para cada valor de frequência de pulsação, foram realizados dois ensaios cada um com diferentes amplitudes de corrente de excitação, a saber: 25 e 50 A.

Os cordões de solda foram realizados a uma distância de $30 \mathrm{~mm}$ do início da chapa, com comprimento aproximado de $240 \mathrm{~mm}$. Com a finalidade de analisar os aspectos geométricos dos cordões de solda obtidos, foram retiradas de cada cordão duas amostras para realização de macrografias e utilizada a média dos valores. A primeira amostra foi retirada a $90 \mathrm{~mm}$ do início do cordão e a segunda, a $130 \mathrm{~mm}$ do início do cordão. Cada amostra passou por um processo de lixamento iniciando com a lixa de 180 mesh e em seguida com as de 220, 320, 400, 600 e 1200 mesh. Após o lixamento as amostras foram polidas com pasta de diamante (com partículas de 0 a $2 \mu m$ ). 0 ataque químico das amostras foi feito com Nital 3,5\% através da técnica de imersão. Com estes procedimentos foi possível revelar 
na amostra as regiões de interesse para a realização das imagens com o auxílio de um estereoscópio. Através do programa open source Image [11] foi possível realizar as medidas de penetração, largura, da área fundida e da área da ZAC dos cordões obtidos.

Com o auxílio do sistema de aquisição SAP V4 foi possível realizar a aquisição dos dados de tensão e corrente da fonte principal, e fazendo uso de um sistema de aquisição baseado num osciloscópio, conforme proposto por Cunha [9], as aquisições da corrente de excitação fornecida pela fonte ultrassônica.

\section{Resultados e Discussão}

Na Figura 4 são apresentas as macrografias da seção transversal dos cordões de solda obtidos com pulsação ultrassônica do arco e sem pulsação (SEM ULT). A priori, conforme pode ser observado, em se tratando dos aspectos dimensionais dos cordões obtidos, percebe-se que a geometria em todos os casos foi semelhante em termos de forma.

Esta geometria semelhante dos cordões vai de encontro ao cálculo da energia de soldagem, podendo-se observar que a energia de soldagem em todos os ensaios foi aproximadamente igual. Foi considerada uma eficiência térmica de $90 \%$ para o processo SAW, pois, segundo Kou [12], a mesma se encontra entre 80 e $99 \%$. Os valores de corrente média e tensão média medidos estão resumidos na Tabela 2. Os valores de corrente média correspondem à soma das correntes fornecidas pela fonte principal e pela fonte ultrassônica.

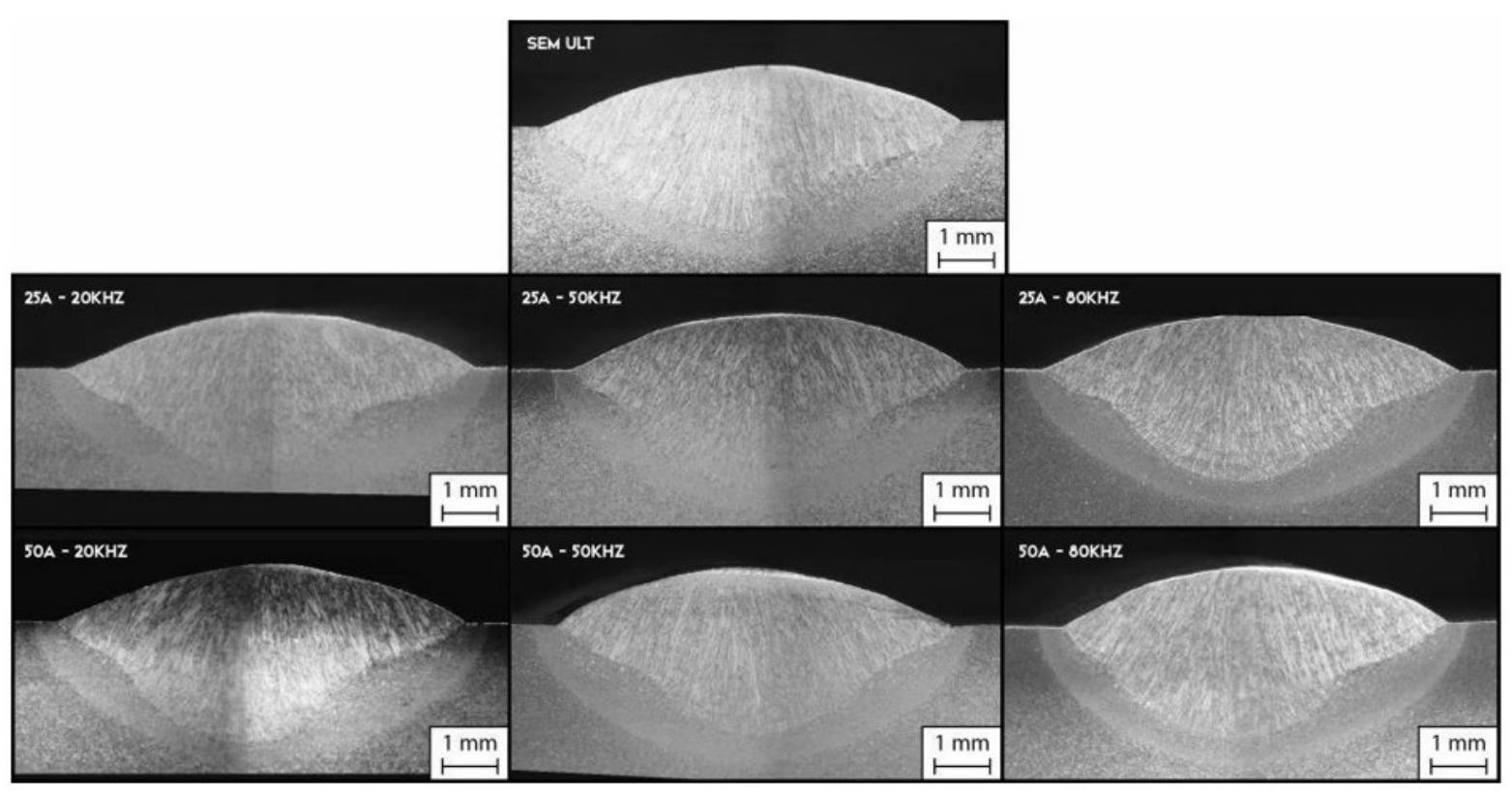

Figura 4. Macrografias da seção transversal dos cordões de solda obtidos nos ensaios.

Tabela 2. Variáveis de soldagem obtidas com a realização dos ensaios.

\begin{tabular}{cccc}
\hline Ensaios $\left(\mathbf{I}_{\text {ultra }} \mathbf{f}_{\text {ultra }}\right)$ & $\begin{array}{c}\text { Tensão média de } \\
\text { arco medida }\end{array}$ & $\begin{array}{c}\text { Corrente média de } \\
\text { soldagem medida }\end{array}$ & $\begin{array}{c}\text { Energia de soldagem } \\
\text { calculada }\end{array}$ \\
$0 \mathrm{~A}-0 \mathrm{kHz}$ & $29 \mathrm{~V}$ & $447 \mathrm{~A}$ & $1167 \mathrm{~J} / \mathrm{mm}$ \\
$25 \mathrm{~A}-20 \mathrm{kHz}$ & $29 \mathrm{~V}$ & $445 \mathrm{~A}$ & $1162 \mathrm{~J} / \mathrm{mm}$ \\
$25 \mathrm{~A}-50 \mathrm{kHz}$ & $29 \mathrm{~V}$ & $452 \mathrm{~A}$ & $1179 \mathrm{~J} / \mathrm{mm}$ \\
$25 \mathrm{~A}-80 \mathrm{kHz}$ & $29 \mathrm{~V}$ & $446 \mathrm{~A}$ & $1165 \mathrm{~J} / \mathrm{mm}$ \\
$50 \mathrm{~A}-20 \mathrm{kHz}$ & $29 \mathrm{~V}$ & $451 \mathrm{~A}$ & $1177 \mathrm{~J} / \mathrm{mm}$ \\
$50 \mathrm{~A}-50 \mathrm{kHz}$ & $29 \mathrm{~V}$ & $442 \mathrm{~A}$ & $1155 \mathrm{~J} / \mathrm{mm}$ \\
$50 \mathrm{~A}-80 \mathrm{kHz}$ & $29 \mathrm{~V}$ & $448 \mathrm{~A}$ & $1170 \mathrm{~J} / \mathrm{mm}$ \\
\hline
\end{tabular}


A média dos valores de penetração dos dois cordões obtidos de cada amostra são apresentados na Figura 5.

Conforme pode ser visto, apenas para a combinação 25 A-20 kHz o valor de penetração foi inferior ao valor obtido sem excitação ultrassônica. Por outro lado, para a combinação 50 A-80 kHz o valor foi superior. Para todos os demais ensaios, os valores de penetração foram aproximadamente iguais àquele adquirido sem excitação ultrassônica. Contudo, ao observarmos os valores obtidos para a frequência de $20 \mathrm{kHz}$, com um aumento da corrente de excitação de 25 para 50 A, há um aumento de aproximadamente $8 \%$ no valor da penetração, ainda que o valor de penetração para a combinação 50 A - $20 \mathrm{kHz}$ seja igual ao valor de penetração sem ultrassom. Um aumento da penetração com o aumento da amplitude de pulsação da corrente também pode ser encontrado para a frequência de $80 \mathrm{kHz}$, ainda que não tão significativo.

Já em relação à largura dos cordões, a diferença entre o menor e o maior valor obtido, foi de 1,1 mm, cerca de 7,5\%, conforme como pode ser observado na Figura 6.

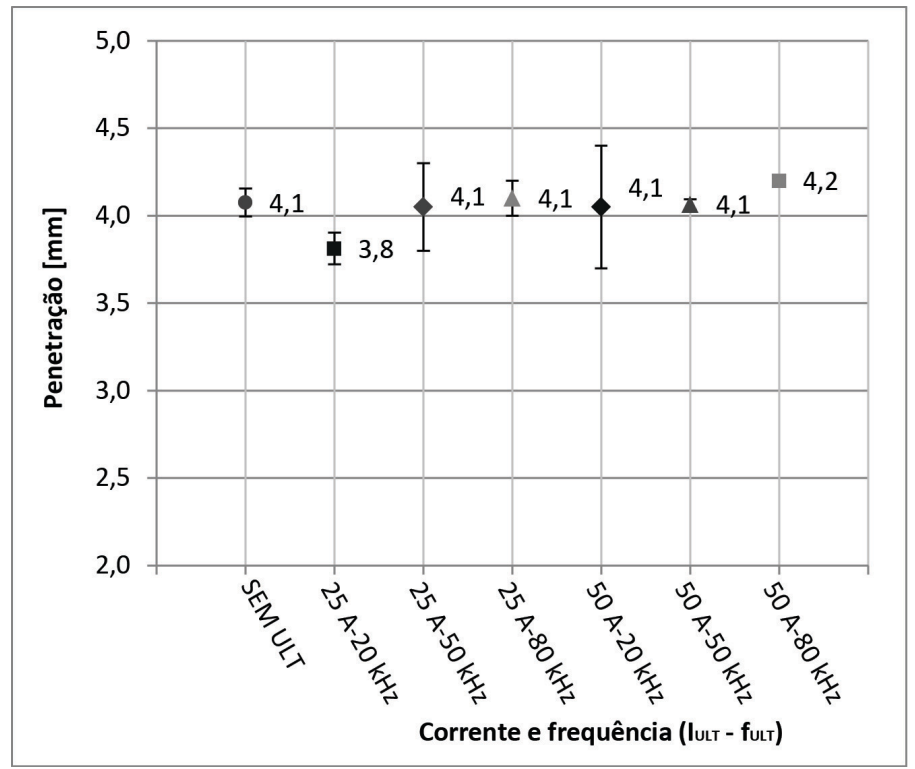

Figura 5. Valor médio de penetração obtido nos cordões de solda realizados.

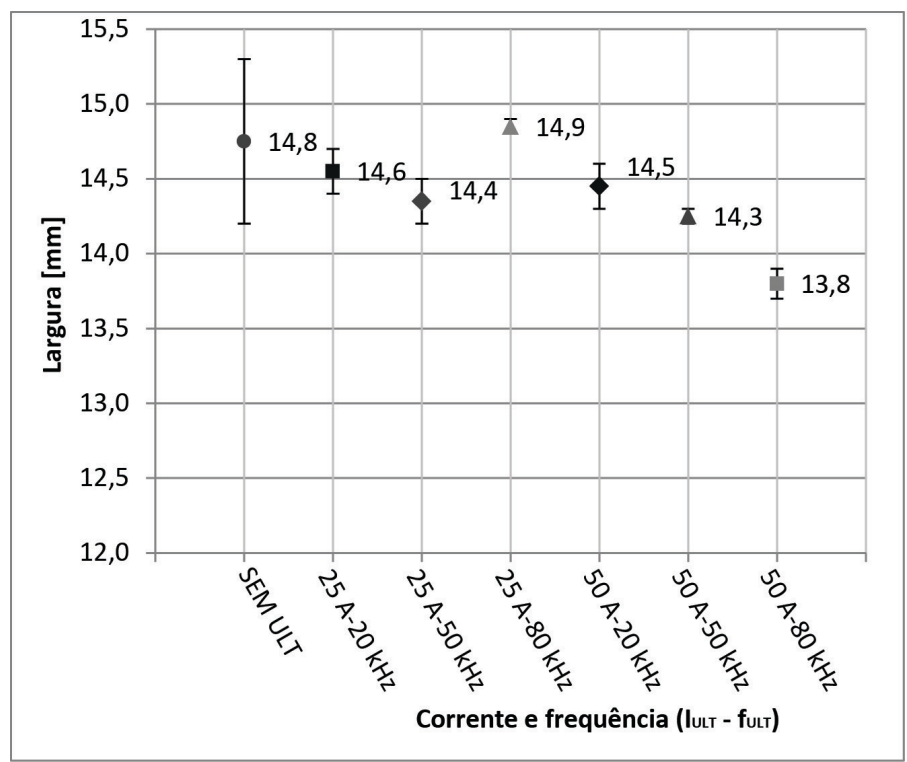

Figura 6. Valor médio de largura obtido nos cordões de solda realizados. 
Ao analisar os resultados da Figura 6 observa-se que apenas para a combinação 25 A-80 kHz o valor de largura obtido foi superior ao encontrado no ensaio sem excitação ultrassônica, ainda que um aumento não expressivo. Os resultados da Figura 6 mostram também que com o aumento da corrente de excitação ultrassônica houve uma diminuição da largura do cordão. Isto, independente da frequência de excitação empregada. Do mesmo modo que para a corrente de excitação de $50 \mathrm{~A}$, o aumento da frequência de pulsação resultou na diminuição na largura. Esta diminuição da largura dos cordões pode estar associada a uma possível constrição do arco relatada na literatura por Qi et al. [13] para o processo TIG. Entretanto, o mesmo não foi observado para a corrente de excitação de $25 \mathrm{~A}$. Isto pode ser devido ao fato de que no processo arco submerso o arco não está livre para oscilar como um transdutor ultrassônico devido a camada de fluxo que o envolve.

Em relação à área fundida, observa-se que para a corrente de excitação de $50 \mathrm{~A}$ o mesmo não se altera em virtude do aumento da frequência de pulsação e seu valor é aproximadamente igual ao valor obtido para o cordão realizado sem excitação (Figura 7).

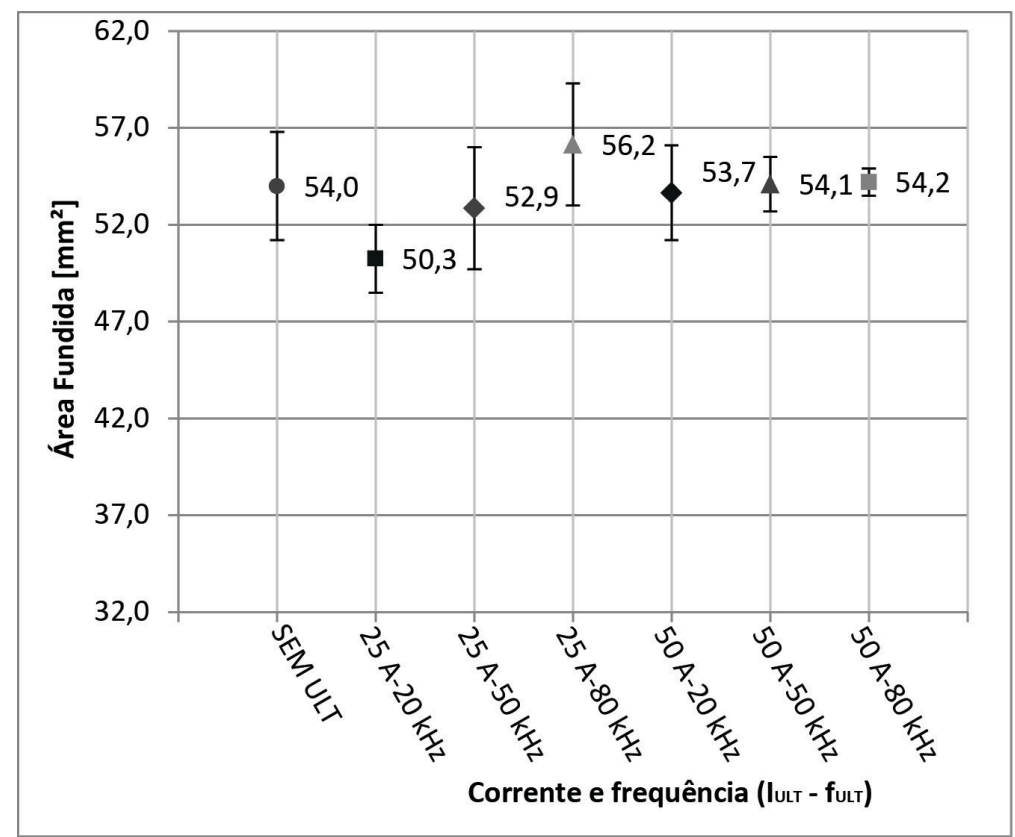

Figura 7. Valor médio de área fundida obtido nos cordões de solda realizados.

Ao analisar os valores de penetração em conjunto com os valores de largura, observa-se que para a corrente de $50 \mathrm{~A}$, a penetração aumentou e a largura diminuiu com o aumento da frequência, resultando cordões de mesma área fundida. Destaca-se ainda a combinação $50 \mathrm{~A}-80 \mathrm{kHz}$ pelo fato de que a mesma resultou na maior penetração e na menor largura de todos os ensaios realizados. Além disso, para esta combinação foi obtida uma área fundida de mesmo valor que o ensaio sem ultrassom indicando, portanto, uma mudança na forma da área fundida, ainda que não expressiva. Por outro lado, para a corrente de $25 \mathrm{~A}$, houve um aumento no valor da área fundida com o aumento da frequência de excitação.

Conforme visto, os possíveis efeitos associados à excitação ultrassônica do arco quanto aos aspectos dimensionais dos cordões realizados, como discutidos, não são expressivos. As variações observadas nas dimensões consideradas nas análises dos cordões não são significativas, de modo que este resultado possa ser devido a outros fatores inerentes à execução dos ensaios e análise. Isto vai contra aos resultados obtidos por Zhang et al. [8] no qual são apresentadas, porém não discutidas, variações significativas de largura, penetração e formato do cordão (Figura 1). Por outro lado, os autores chamam a atenção para o aumento significativo do tamanho da zona afetada pelo calor com o aumento da frequência de excitação. Com base nisso, neste trabalho foram avaliadas também as dimensões da ZAC obtidas com os ensaios realizados (Figura 8). 


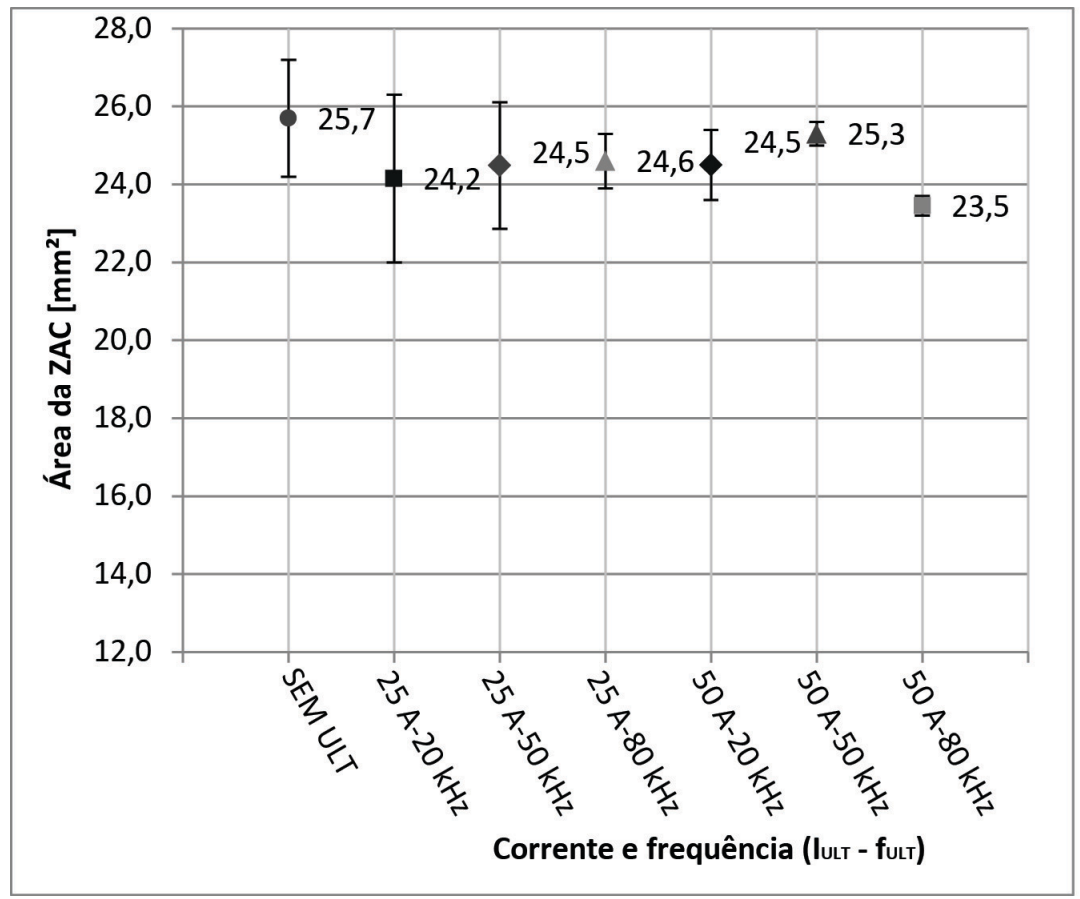

Figura 8. Valor médio de área da ZAC obtido nos cordões de solda realizados.

De forma contrária aos resultados obtidos por Zhang et al. [8], foi obtida uma área da ZAC inferior àquela produzida sem excitação da corrente, em especial para a combinação 50 A-80 kHz. Para esta combinação a área fundida obtida foi a mesma do cordão sem ultrassom, com mesma forma e com uma redução da ordem de $11 \%$ da área da ZAC em relação ao cordão sem ultrassom. Este resultado corrobora a afirmação de Zhang et al. [8] de que o ultrassom acelera a condução de calor. Contudo, estes mesmos autores justificam o aumento da ZAC com base no efeito do ultrassom na direção do eixo axial do arco. Neste sentido tal efeito é mais intenso devido a maior amplitude de vibração ultrassônica no mesmo, o que promove o alongamento da ZAC nesta direção. Entretanto este comportamento da ZAC obtido por estes autores é típico de um aumento do aporte de calor à peça. Visto que não são apresentados em seu trabalho maiores detalhes a respeito das variáveis utilizadas, este resultado de aumento da ZAC e mudanças dimensionais do cordão sugerem que a energia de soldagem utilizada nos ensaios não foi a mesma. Logo, o aumento da ZAC obtido pelos mesmos pode não estar vinculado à pulsação da corrente em frequências ultrassônicas.

Apesar das mudanças encontradas na geometria do cordão não serem significativas para o processo SAW, para o processo TIG é possível obter um aumento da penetração e largura com a aplicação da técnica. Utilizando uma corrente principal de $80 \mathrm{~A}$ e uma corrente de excitação ultrassônica de $50 \mathrm{~A}$, variando a frequência de pulsação também entre 20 e 80 kHz, Cunha [9] não encontrou uma correlação direta entre a frequência e a largura e penetração do cordão de solda. Contudo, em todos os ensaios realizados com pulsação ultrassônica com forma de onda retangular, os valores de penetração e largura obtidos foram superiores àqueles obtidos com TIG com corrente constante. Considerando um valor médio de penetração e largura, em relação ao TIG CC, houve um aumento de $10 \%$ na largura e 33\% na penetração. Esse aumento expressivo, sobretudo na penetração, obtido no processo TIG, pode ser atribuído à relação entre a corrente ultrassônica e a corrente principal utilizada $\left(I_{\mathrm{ULT}} / \mathrm{I}_{\mathrm{PRI}}\right)$, de $50 \mathrm{~A} / 80 \mathrm{~A}$. Acredita-se que esta relação muito próxima de um atua no sentido de provocar uma oscilação mais significativa do arco. Por outro lado, no presente trabalho, esta relação foi de 50 A/450 A, assim, com uma relação de cerca de dez vezes menor acredita-se que o arco não tenha condições de oscilar tão facilmente devido a sua rigidez imposta pela alta corrente principal de soldagem (450 A). Além disso, assim como mencionado, há ainda de ser considerada a presença do fluxo depositado sobre o arco, que tende a atuar como uma barreira mecânica contra a oscilação do mesmo. Estes poderiam se constituir em fatores que conduziram a não observância de mudanças expressivas na geometria do cordão, tampouco nos valores obtidos de largura e penetração em relação 
ao ensaio sem pulsação ultrassônica, diferente do que ocorreu no processo TIG. Apesar das mudanças na geometria do cordão encontradas por Cunha [9], o autor não conseguiu estabelecer uma relação plausível entre os aspectos dimensionais dos cordões e as variáveis relativas à corrente de excitação ultrassônica, resultado esse semelhante ao obtido no presente trabalho para o processo arco submerso.

\section{Conclusões}

Com base nos resultados obtidos, é possível concluir que:

- Não foram observadas mudanças significativas em relação aos aspectos dimensionais dos cordões obtidos com a aplicação da técnica de excitação ultrassônica do arco para as combinações de corrente e frequência de pulsação investigadas;

- Não foi possível estabelecer uma relação consistente entre os aspectos dimensionais dos cordões e as variáveis de corrente de excitação ultrassônica;

- Empregando correntes de pulsação de $50 \mathrm{~A}$, foi possível obter, para todas as frequências investigadas, uma diminuição da área da ZAC. Em especial para a combinação 50 A-80 kHz onde esta redução foi mais expressiva, cerca de 11\%;

- Na frequência de pulsação de $20 \mathrm{kHz}$, um aumento na corrente de excitação de $25 \mathrm{~A}$ para $50 \mathrm{~A}$ resultou num aumento de $8 \%$ na penetração.

\section{Agradecimentos}

Os autores agradecem ao Laboratório de Tecnologia da Soldagem (LTS) da UFSC Joinville pela oportunidade de utilização dos equipamentos e do suporte. Os autores estendem ainda os agradecimentos ao PET EMB pelo apoio financeiro na compra dos consumíveis e ao Instituto SENAI de Inovação em Sistemas de Manufatura pelo auxílio na realização das análises.

\section{Referências}

[1] Wu M, Wang Z, Li L, Sun D, Duan X. Study on mechanism of arc-excited ultrasonic. Chinese Journal of Mechanical Engineering. 1999;12(1):1-3.

[2] Lei YC, Wang ZW, Chen XZ. Effect of arc-ultrasound on microstructures and mechanical properties of plasma arc welded joints of SiCp/Al MMCs. Transactions of the China Welding Institution. 2011;21(2):272-277.

[3] Lei Y, Xie W, Li C, Chen X. Numerical analysis of the molten pool vibration mode for arc-ultrasonic keyhole plasma arc welding. China Welding. 2012;21(1):22-26.

[4] Zhou S, Zhao H, Qi B. Ultra-sonic pulse tungsten inert-gas arc welding of Ti-6Al-4V alloy. Transactions of the China Welding Institution. 2010;10:15.

[5] He LB, Hao HW, Li LM, Wen XW, Wu MS. Effect of arc-ultrasonic on welding structure and property of Q235A steel with SMAW. Hot Working Technology. 2006;1.

[6] Yang BY, Hao HW, Li LM, Wu MS, Wang S. Study on arc-ultrasonic saw improving structure and property of 09MnNiDR welding joint. Hot Working Technology. 2006;2.
[7] Wu M, Zhang Y, Li L, Hao H. Arc-excited ultrasonic welding to improve the quality of 09MnNiDR joints. Journal of Tsinghua University. 2006;2:161-164.

[8] Zhang C, Wu M, Du J. Improving weld quality by arc-excited ultrasonic treatment. Tsinghua Science and Technology. 2001;6(5).

[9] Cunha TV. Desenvolvimento e avaliação de tecnologia para soldagem TIG com pulsação ultrassônica [tese de doutorado]. Florianópolis: Programa de Pós-graduação em Engenharia Mecânica, Universidade Federal de Santa Catarina; 2013.

[10] Zhang C, Wu M, Hao H, Han Z. Research on resonance mechanism of arc-ultrasonic. Journal of Materials Science and Technology. 2000;16(5):525-528.

[11] ImageJ [página da internet]. 2015 [acesso em 20 ago. 2015]. Disponível em: http://imagej.net/

[12] Kou S. Welding metallurgy. 2. ed. New York: John Willey \& Sons; 2003. p. 33.

[13] Qi BJ, Yang MX, Cong BQ, Liu FJ. The effect of arc behavior on weld geometry by high-frequency pulse GTAW process with 0Cr18Ni9Ti stainless steel. International Journal of Advanced Manufacturing Technology. 2012; 66(9):1545-1553. 